\title{
Balancing axial force in centrifugal pumps with pump out vanes
}

\author{
Marek Szlaga ${ }^{1}$ \\ ${ }^{1}$ Institute of Heat Engineering, Warsaw University of Technology. 25 Nowowiejska Street, Warsaw 00-665, szlagamarek@gmail.com, \\ Poland
}

\begin{abstract}
Application of pump out vanes is one of the solutions to reduce hydraulic axial force generated during centrifugal pump operation. This article presents the cause of the occurrence of hydraulic axial force and method of calculating pressure distribution at the rear of the impeller used to design pump out vanes properly. It illustrates results of pump out vanes CFD calculations and its validation by measurements. The article reviews the methods of reducing pressure on the rear wall of the centrifugal pump's rotor using pump out vanes. It presents empirical formulae allowing calculation of pressure depending on the geometrical parameters of the blades. The article presents various design solutions of pump out vanes.
\end{abstract}

\section{NOMENCLATURE}

\begin{tabular}{|c|c|c|}
\hline$c_{w l}$ & {$[\mathrm{~m} / \mathrm{s}]$} & absolute velocity \\
\hline $\begin{array}{l}c_{f, w} \\
\mathrm{imp}\end{array}$ & $\begin{array}{l}{[-]} \\
\text { rroud }\end{array}$ & friction coefficient of casing and \\
\hline$k$ & {$[-]$} & rotation factor \\
\hline$Q_{t h}$ & {$\left[\mathrm{~m}^{3} / \mathrm{s}\right]$} & theoretical pump capacity \\
\hline$\omega$ & {$[1 / s]$} & angular velocity \\
\hline$\rho$ & {$\left[\mathrm{kg} / \mathrm{m}^{3}\right]$} & density \\
\hline
\end{tabular}

\section{Introduction}

The rotational movement of the pump creates a complex pressure field around the impeller. As a result, during the pump operation, the impeller is subjected to radial and axial forces. The ability to calculate these forces is necessary for the correct design of the shaft and sizing pump bearing system.

Many different methods and solutions are used to balance the hydraulic axial force. How to deal with axial thrust depends on the type of pump, its design, size, and its rotational speed. In pumping devices of small size, operating at low speeds, all axial thrust can be absorbed by the capacity of thrust bearings since the magnitude of this force is small. Larger pumps operating at high speeds may already require the use of specially designed balancing systems. In multi-stage pumps balance disk or balance piston is used. For pumps with a double-entry impeller, the symmetry of impeller reduces axial thrust. In single-stage pumps, two methods of reducing the axial force are most often used: using pump out vanes located on the rear shroud or using an annular sealing ring with balance holes on the rear shroud.

In the impeller sidewall gaps, at the walls of the rotating impeller, there are radially outward fluid flows caused by inertial forces acting on the liquid by the rotor. For reasons of continuity fluid flows radially inwards at the walls of the stationary casing (Fig. 1). The occurrence of leaks (e.g. at the annular seal on the front shroud) resulting from the pressure difference on both sides of the seal also has a very large impact on the velocity field in these areas. Depending on the pump design, leaks can also occur in the space behind the impeller (including relief holes, shaft seal, multi-stage pumps). The effect of these phenomena is the presence of complex, three-dimensional flow phenomena in the impeller sidewall gaps.

As a consequence, there are different pressure distributions on front and rear impeller shroud (Fig. 2), which is the main reason for the hydraulic axial force in the pumps.

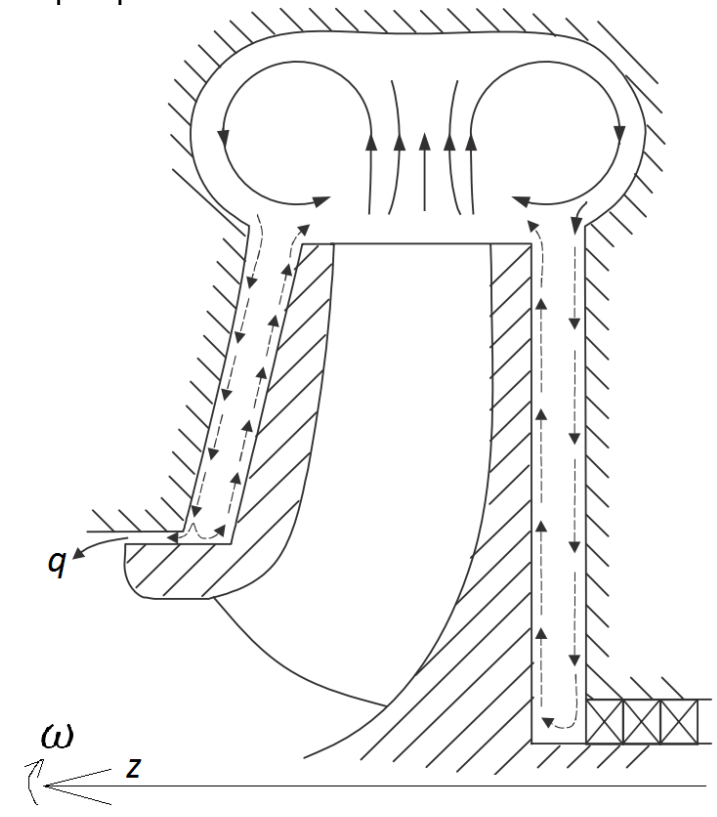

Fig. 1. Velocity distribution in the impeller sidewall gaps [5] 


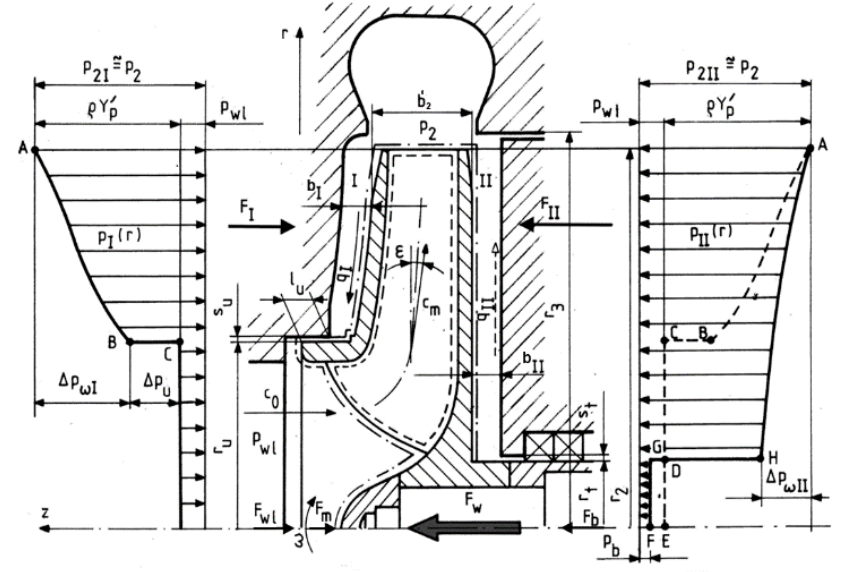

Fig. 2. Pressure distribution and axial forces on an impeller of a single-stage pump [4]

\section{Axial thrust calculation}

Assuming that the pressure distributions are axially symmetrical, which based on many research carried out has been proven for operation at the nominal pump capacity, the forces $F_{I}$ and $F_{I I}$ acting on the surfaces of the front and rear impeller shrouds can be determined based on the following equation

$$
\begin{aligned}
& F_{I}=2 \pi \int_{r_{u}}^{r_{2}} p_{I}(r) r d r \\
& F_{I I}=2 \pi \int_{r_{t}}^{r_{2}} p_{I I}(r) r d r
\end{aligned}
$$

Where $r_{2}$ is the impeller outlet radius, $r_{u}$ is the annular seal radius on the front shroud, $r_{t}$ is the impeller hub radius.

The other forces acting on the impeller are:

- force resulting from the pressure at the impeller inlet $p_{w l}$

$$
F_{w l}=\frac{\pi}{4} d_{u}^{2} p_{w l}
$$

- force from atmospheric pressure $p_{b}$ acting on the pump shaft

$$
F_{b}=\frac{\pi}{4} d_{t_{-} \text {shaft }}^{2} p_{b}
$$

- reaction force resulting from changing the flow direction in impeller by $90^{\circ}$ from axial to radial

$$
F_{Q}=\rho \frac{Q_{t h}^{2}}{A_{w l}}=\rho Q_{t h} c_{w l}
$$

Net hydraulic axial force acting on the rotor results from the sum of the listed forces, including their directions

$$
F_{w}=F_{I I}-F_{I}-F_{w l}-F_{Q}+F_{b}
$$

\section{Fluid rotation factor}

Based on many research, it can be concluded that across areas I and II, the pressure practically does not change, that means

$$
\frac{\partial p}{\partial z} \cong 0
$$

Momentum equation in the radial direction takes the form

$$
\frac{\partial p}{\partial r}=\rho \frac{v_{\varphi}^{2}}{r}
$$

To determine the pressure distribution in the in the impeller sidewall gaps the velocity $v_{\varphi}$ is needed with which the fluid is rotating. Assuming that pressure distributions $p_{I}(r)$ and $p_{I I}(r)$ depend only on the local fluid rotation

$$
k_{I, I I}(r)=\left(\frac{v_{\varphi}}{\omega r}\right)=\left(\frac{\omega_{c l, I I}}{\omega}\right)
$$

For impeller sidewall gaps following equation can be written based on (8) and (9)

$$
\frac{1}{\rho} \frac{\partial p_{I, I I}}{\partial r}=\frac{v_{\varphi}^{2}}{r}=\omega_{c}^{2} r=k_{I, I I}^{2}(r) \omega^{2} r
$$

To calculate the net hydraulic axial force and obtain pressure distributions in the I and II areas of the impeller, a rotation factor is needed as $k=k(r)$.

Many experimental and theoretical studies show that the circulation coefficient is a complex function of the geometrical dimensions of the flow area, roughness and the pump operating parameters.

In the vast majority of cases, the flow in the sidewall areas is turbulent. Rotation factor can be determined by numerically solving the Reynolds equations. For the purposes of preliminary calculations of the hydraulic axial force in the pump design process, these relationships are too complicated. However, the assumption that $k_{I}=k_{I I}=0,5$ used e.g. in books [1,2] is too simplified, which generates errors in calculating the forces acting on the rotor reaching several tens of per cent [3]. This approach does not take into account the complexity of the flow phenomenon in the impeller sidewall areas.

Jedral [4] suggests to use the following values of rotation factor depending on the net flow through the impeller sidewall gap:

- if the leakage is directed radially outwards $k=0,3 \div 0,4$,

- if the leakage is directed radially inwards $k=0,7 \div 0,9$,

- for lack of the leakage $(q=0) k=0,45 \div 0,5$.

For the purpose of rotation factor calculation Gulich [5] suggests using the following equation when there is no net through-flow

$$
k=\frac{1}{1+\left(\frac{r_{3}}{r_{2}}\right)^{2} \sqrt{\left(\frac{r_{3}}{r_{2}}+5 \frac{t_{a x}}{r_{2}}\right)^{c_{f, w}} \frac{c_{f, R}}{c_{f, 2}}}}
$$

Where $t_{a x}$ is the cylindrical part of the casing with $r_{3}$ radius in the area of sidewall gap.

In [5] a separate procedure can also be found to calculate the circulation rate in case of net flow through the impeller sidewall gap.

For averaged $k_{I}$ and $k_{I I}$ values, after integrating the equation (10), pressure distributions can be obtained in the areas in front and behind the rotor

$$
p_{I}(r)=p_{I 2}-\rho \omega^{2} \int_{r_{u}}^{r_{2}} k_{I}^{2}(r) r d r
$$




$$
p_{I I}(r)=p_{I I 2}-\rho \omega^{2} \int_{r_{t}}^{r_{2}} k_{I I}^{2}(r) r d r
$$

After inserting formulae for pressure distribution into formulae (1) and (2), the force $F_{I}$ i $F_{I I}$ can be calculated.

\section{Balancing axial force with expeller vanes}

Among the forces acting on the impeller, the largest component is the force $F_{I I}$ acting on the rear shroud. To reduce the hydraulic axial force, $F_{I I}$ must be reduced by reducing the pressure in the area of rear shroud. In singlestage pumps for this purpose expeller vanes on the rear impeller shroud can be used. Pump out vanes increase the rotation factor of the liquid and, as a result, reduce the pressure acting on the impeller in this area (area $\mathrm{ABC}$ as shown in Fig. 3).

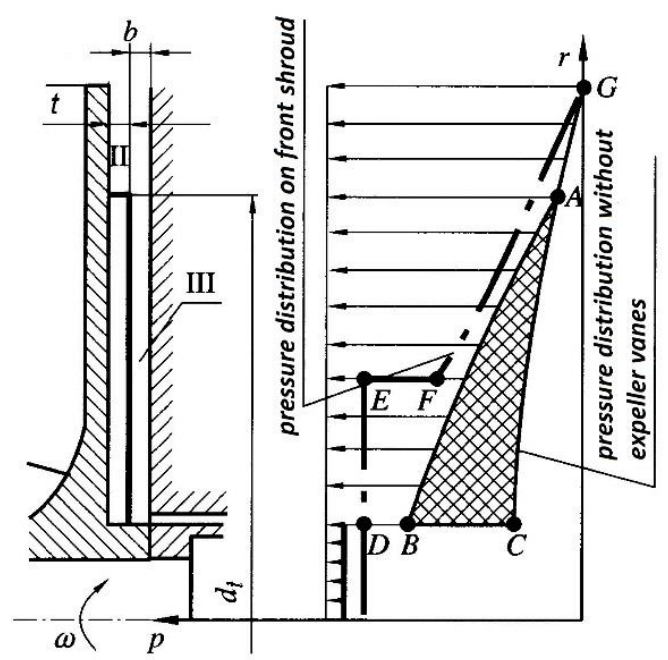

Fig. 3. Pressure distribution for the impeller with expeller vanes[4]

The advantages of reducing axial force with pump out vanes are:

- reducing the amount of pump parts (reduced production costs and subsequent repairs) - expeller vanes are the least costly design,

- easier standardization of rear cover since no seal,

- stuffing box protection when pumping solids contaminated fluids,

- the possibility of completely balancing the axial force over a wide range of performance and inlet pressures.

Expeller vanes are sometimes used also on the front impeller shroud. In this solution, their purpose is not to reduce the net axial force, but to protect the annular seal on the front shroud against solids entering the impeller sidewall gap.

These advantages make the expeller vanes widely used for single-stage pumps for pumping water, mixtures of liquids and solids, aggressive fluids and as chemical pumps.

The use of expeller vanes increases the disk friction losses and, as a result, reduces the efficiency of the pumps. Oversizing of pump out vanes in extreme cases can cause a change in the direction of the axial force and its increase in relation to the operation without them. Considering these aspects, the use of expeller vanes require accurate calculation formulae.

Various equations and approaches can be found in the literature for determining the value of the rotation factor when using expeller vanes. They differ in the scope of application and the number of simplifying assumptions. Lazarkiewicz and Troskolański [1] suggest using 4 to 6 vanes with a height of 4 to $7 \mathrm{~mm}$ and very small gaps between blades and the casing. Authors assume that the liquid between expeller vanes rotates at a constant angular velocity equal to the impeller speed. If expeller vanes are trimmed $\left(d_{t}<d_{2}\right)$, authors assume that the fluid rotation factor in the plain outer part of the rear shroud decreases immediately up to $k=0.5$.

Stepanoff [6] assumed that the fluid rotation coefficient between expeller vanes and the fixed wall of the housing does not change along the radius and expressed it by the formula

$$
k=0,5\left(1+\frac{t}{t+b}\right)
$$

Where $t$ and $b$ are shown in Fig. 3. In the plain outer part of the rear shroud, the rotation coefficient changes rapidly to a value of $k=0.5$.

Gulich [5] proposes a formula determining the value of the circulation coefficient for a disc bladed through the following equation

$$
k_{t}=\frac{1}{1+0,13 \frac{t}{t+b} \sqrt{\frac{r_{2}}{t i}}}
$$

For trimmed expeller vanes $\left(d_{t}<d_{2}\right)$ following equation is used to determine the average value of the rotation coefficient

$$
k_{s r}=\sqrt{\left(\frac{d_{t}}{d_{2}}\right)^{\left(2-0,9 \frac{d_{t}}{d_{2}}\right)}\left[k_{t}^{2}-k^{2}\right]+k^{2}}
$$

In [5] following recommendations for the design of vanes can be found:

- number of expeller vanes equal to number of impeller blades,

- shape of expeller vanes same as of impeller blades (mostly back swept),

- outer diameter: $d_{t}=0,85 d_{2}$,

- vane height: $t / r_{2}=0,04$ to 0,05 ,

- axial clearance $b / r_{2}=0,01$,

- width of expeller vanes equal to height $h$ or equal to nominal blade thickness (whatever is greater).

As part of the research work carried out at Institute of Heat Engineering, measurements of pressure distribution on the bladed rotating disc were carried out (rotating disc is marked with number 8 in Fig. 4). During tests, pressures on several disc diameters were measured, and then local average circulation coefficient values were determined based on the following equation:

$$
k_{i}=\frac{30}{\pi n} \sqrt{\frac{2\left(p_{i+1}-p_{i}\right)}{\rho\left(r_{i+1}^{2}-r_{i}^{2}\right)}}
$$


The radius of the rotating disc on the stand was $r_{2}=160 \mathrm{~mm}$, and the radius of the cylindrical casing $r_{3}=172,5 \mathrm{~mm}$.

Following blade radii were examined $r_{t}=160 ; 140 ; 120 ; 100 ; 80 \mathrm{~mm}$, blade height $t=4 ; 6,3 ; 8 ; 10 \mathrm{~mm}$. Axial clearance $b=1 ; 2 ; 4 ; 8 \mathrm{~mm}$. Discs with different number of blades were tested; $i=4 ; 8 ; 12 ; 16$ at rotational speeds $n=2250 ; 1500 ; 750 \frac{1}{\min }$ and the net flow through the rotating domain $Q_{s}=0 ; 0,3 ; 0,6 \frac{\mathrm{m}^{3}}{\mathrm{~h}}$.

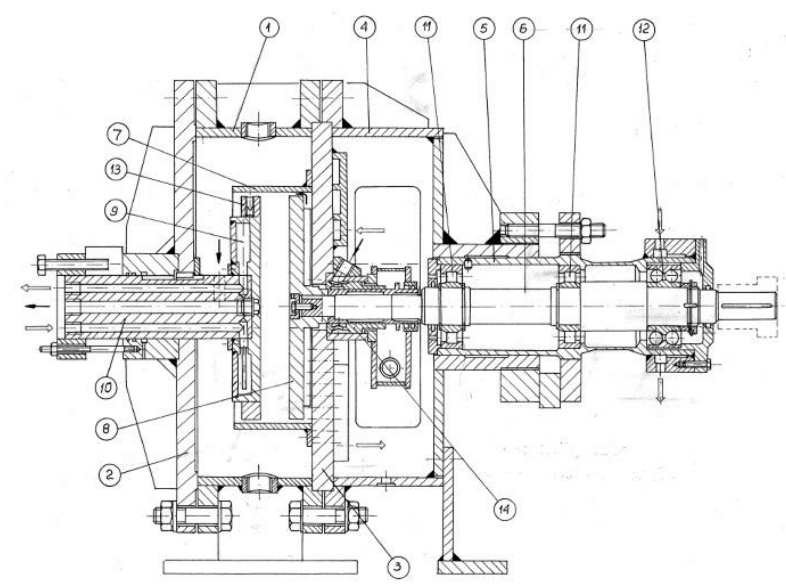

Fig. 4. Sectional drawing of the test stand

Selected measurement results were compared with results obtained by the author of numerical simulations performed in the Ansys Fluent software. The chart below shows the rotation coefficient obtained on the basis of measurements with values obtained on the basis of numerical calculations and calculated from formulae found in the literature.

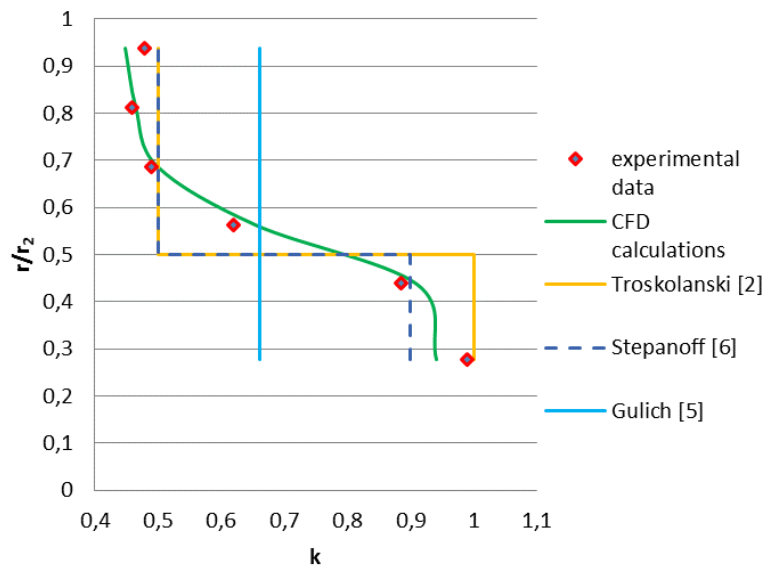

Fig. 5. Rotation factor for $r_{t}=80 \mathrm{~mm} ; t=4 \mathrm{~mm}$; $b=1 \mathrm{~mm} ; i=12 ; n=2250 \frac{\mathrm{obr}}{\min } ; Q_{s}=0$

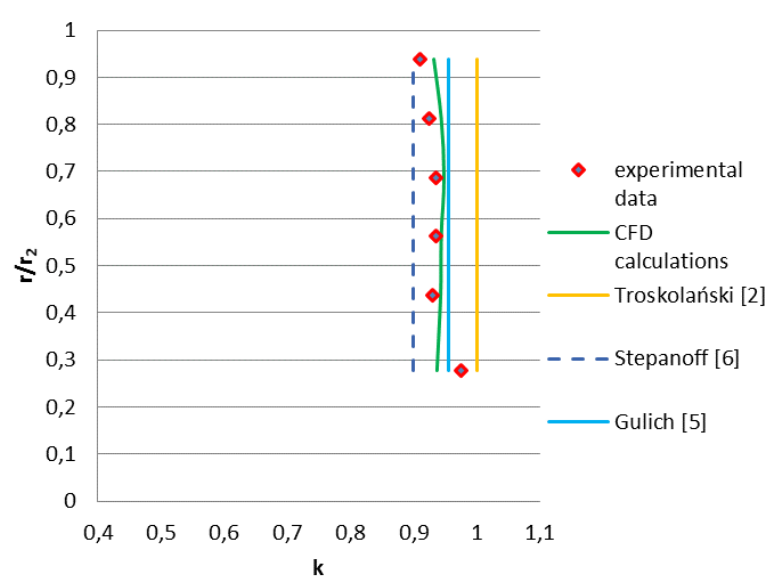

Fig. 6. Rotation factor for $r_{t}=160 \mathrm{~mm} ; t=4 \mathrm{~mm}$; $b=1 \mathrm{~mm} ; i=12 ; n=2250 \mathrm{obr} / \mathrm{min} ; Q_{s}=0$

Comparing measurements results and CFD calculations with the formulae found in the literature, there is a very large discrepancy in the case of trimmed vanes. The model of the step change of the rotation factor at the transition from the area with vanes to the flat disc turns out to be completely inadequate. The rotation factor changes gradually and begins to decrease already in the area of occurrence of the vanes. In no case was $k=1$ reached.

In the case of a fully bladed disc, test results are close to calculated values. CFD calculations allow to determine the pressure distribution with much greater accuracy than empirical formulae.

In [5] information can be found that in the case of expeller vanes, a solution generating lower energy losses in relation to straight radial blades is the use of curved blades. Such solutions can be found in manufactured pumps (Fig. 7).

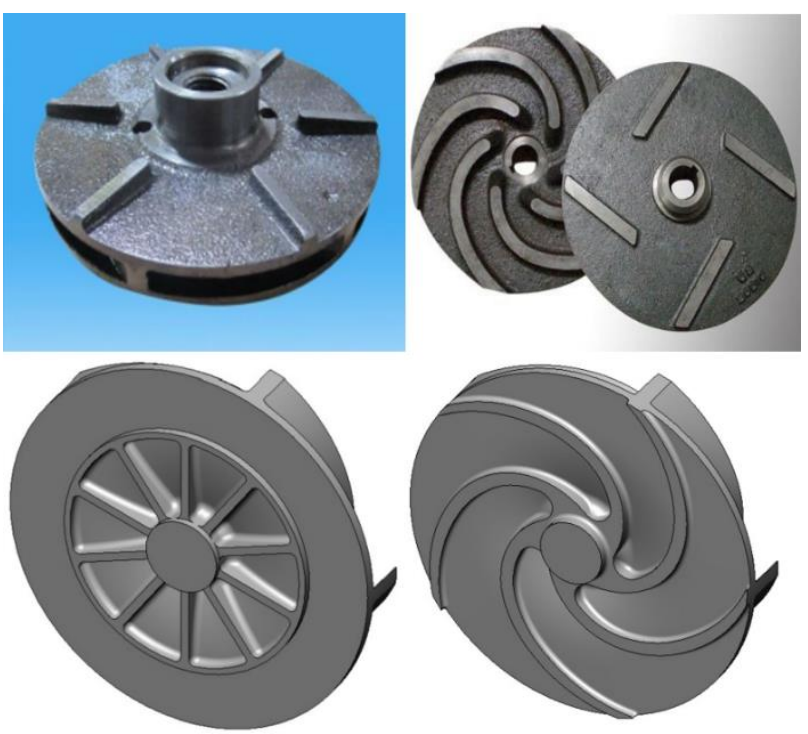

Fig. 7. Different solutions in design of expeller vanes

There is a lack of literature confirming this thesis and presenting the impact of other types of unloading blades (other shapes, positioning, angles) on the rotation factor and disk friction losses. It also marks a certain area of 
solutions and research that should be addressed in scientific works. The goal of further research should be to create guidelines and mathematical equations that allow designing optimal unloading blades in terms of effectiveness in reducing hydraulic axial force and minimizing energy losses.

\section{Conclusions}

Expeller vanes are the simplest and the least expensive solution in case the reduction of axial thrust is required. For expeller vanes design, accurate formulae shall be used to diminish the increase of disk friction losses.

This paper presents several formulae used to determine the rotation factor in the impeller sidewall gaps in case pump out vanes are used. Selected formulae for rotation factor calculation were compared with experimental data and CFD calculations. Significant discrepancies were found especially for trimmed expeller vanes.

Further research should be carried out to determine the impact of expeller vanes shape and positioning on rotation factor and disk friction losses.

\section{References}

1. Pfleiderer C.: Die Kreiselpumpen fur Flüssigkeiten und Gase, 5. Aufl, Springer, Berlin, 1961.

2. Troskolański A.T., Łazarkiewicz S., Pompy wirowe, WNT, Warsaw (1973).

3. Jedral W., Metoda obliczania sit wzdtużnych $w$ pompach wirowych, Przegląd Mechaniczny, Vol. 13, pp. $11 \div 14$ (1992).

4. Jedral W., Rotodynamic Pumps, PWN, Warsaw (2001).

5. Gülich J.F., Centrifugal Pumps, Springer-Verlag, Berlin Heidelberg (2014).

6. Stepanoff A.J., Centrifugal and axial flow pumps. Theory design and application, J. Wiley Sons Inc., New York (1957). 